\title{
ON THE POSSIBLE RELATIONSHIP BETWEEN THE PHOTOMETRIC PARAMETERS OF THE AGB STARS AND THEIR EVOLUTIONARY STATUS
}

\author{
L.S. KUDASHKINA and I.L. ANDRONOV \\ Department of Astronomy, Odessa State University, \\ T.G.Shevchenko Park, Odessa 270014 Ukraine
}

The statistical study of the Long-Period Variables, provided by various authors, show, that there is a relationship between the photometric characteristics and the physical properties. For example, the asymmetry of the light curve may be related with the strength of the shock wave. The presence of the maser-fed line emission characterizes the definite evolutionary stage of the Long-Period Variable.

In this work, we present the results on the Mira - type stars with the periods $\mathrm{P}<600$ days, which may be subdivided into 4 groups according to the value of $\mathrm{P}$ :
1) $P<200$ days,
2) $200<P<300$ days,
3) $300<P<400$ days,
4) $P>400$ days.

The stars from the groups 2 and 3 have the most stable light curves with the significant asymmetry, but the stars from the group 3 are often observed as the maser sources.

The following photometric characteristics are studied: the amplitude, the asymmetry, the variability of the maximum brightness, the period and its stability, the presence and the duration of the hump at the ascending branch of the light curve, as well as the spectral class. For the group of the Mira-type stars, the light curves published by the AAVSO members were digitized, and the best fit period and the corresponding Fourier coefficients were computed and converted into the amplitudes and phase differences. The optimum number of the harmonics varies in the range from 1 to 7 for the different stars.

One of the aims is to choose the criterium, which characterizes the evolutionary stage of the Long-Period Variable, and is based on the observational characteristics (photometry and polarimetry in the optical and IR bands, spectral properties, maser emission). The various correlations between the photometric parameters are studied for the individual stars (cycle-to-cycle variations), as well as for the groups. 